
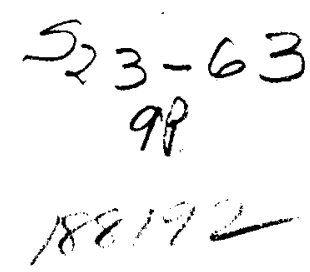

\title{
Algorithms for Adaptive Control of Two-Arm Flexible Manipulators Under Uncertainty
}

\author{
J.M. Skowronski \\ University of Queensland \\ St. Lucia, Australia 4067
}

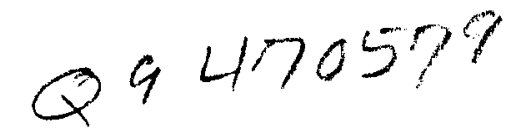

1. Abstract

$i: \ldots$. chl

The paper a nonlhear extension of model reference adaptive control (MRAC) techñque/tis guide a double arm nonlinearizable robot manipulator with flexible links, driven by actuators collocated with joints subject to uncertain payload and inertia. The objective is to track a given simple linear and rigid but compatible dynamical model in real, possibly stipulated time and within stipulated degree of accuracy of convergence whilc avoiding collision of the arms. The objective is attained by a specified signal adaptive feedback controller and by adaptive laws, beth given in closed form. A case of 4 DOF manipulator illustrates the technique.

\section{Introduction}

The MRAC technique becomes popular proposition for guidance of recent robot manipulators, with demand for preciston pointing in difficult conditions, under the action of full scale dynamic forces, and subject to uncertainty in parameters. Such manipulators, particularly these used on spacecraft are highly nonlinear and nonlinearizable structures (geometric nonlinearity of elastic links, large angle articulation, nonlinear coupling of DOF's, nonignorable gyro and Coriolis forces, several equilibria). while classical MRAC is 1 inear and applicable to rigid bodies only. Thus the extension is needed for handling nonlinearity, see [1], and flexible links, see $[2]$. On the other hand many robotic objectives, again particularly these in difficult space conditions require at least two arm systems. Thus the tracking has to be a double MRAC (mutual reference adaptive control) which secures tracking the same model by two arms while avoiding mutual collision - cf. [3], [4]. If adaptive (self-organizing) control is intended, the tracking relates not to a given path but to a given dynamic targetmodel with prescribed target-parameters. We take the model simple thus rigid and 1 inear, but locally compatible with the nonlinear $3 \mathrm{rms}$ regarding equilibria. Each arm is represented as an open chain with $n$ DOF, nonlinear characteristics and coupling, elastic links, driven by actuators collocated with joints, under uncertain inertia parameters and uncertain payload. The tracking is done in real possibly stipulated time by a designed signal adaptive feedback controller and integrable adaptive laws in the state space, while avoiding collision between arms of all the joints (and elastic nodes) in Cartesian configuration space.

\section{Motion Equations}

l.agrange motion equations give the rigid dynamics of the arms in the general format

$$
A^{j}\left(q^{j}, s^{j}\right) \ddot{q}^{j}+\Gamma^{J}\left(q^{j}, \dot{q}^{j}, \lambda^{j}\right)+\Pi^{j}\left(q^{j}, \lambda^{j}, s^{j}\right)=B^{j}\left(q^{j}, \dot{q}^{j}\right) u^{j}, j=1,2 \text {. }
$$

where $q^{j}(t) \cdot J_{q} \cdot \mathbf{R}^{n}, t: t_{0}=0$, is the configuration vector of the joint variables $q_{l}^{j}, \ldots, q_{n}^{j}$ of the $j$-th arm varying in the known bounded work region $a_{q}$ of the configuration space $R^{n} ; \dot{q}(t)$ is the corresponding vector of joint velocities in the specified bounded subset $A_{\dot{q}}$ of the space tangent to $R^{n}$;

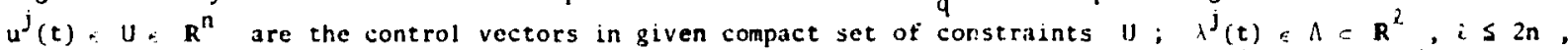
are the vectors of adjustable system parameters in bounded bands of values $\Lambda$, and $s^{j}(t) \in S \in R^{k}$ is an uncertainty parameter within the known band $s$. Moreover $f^{j}\left(q^{j}, s^{j}\right)$ are the inertia n×n matrices obtained in the known way from the quadratic form of kinctic energy. The vectors $11{ }^{j}=\left(\pi l_{1}^{j}, \ldots, n_{n}^{j}\right)^{T}$ represent potential forces (gravity, spring) while ${ }^{j}=\left(i_{1}^{j}, \ldots, n_{n}^{j}\right)^{T}$ represent the internal nonpotential acting forces (Coriolis. gyro, centrifugal, damping structural or viscous, etc.) and ${ }_{B}{ }^{j}$ is the actuator transmission (gear) nonsingular $n m$ matrix. The control vectors $u j(t)$ are selected for the objectives of tracking and avoidance by adaptive feedback control programs $u^{j}(t)=p^{j}\left(o^{l}(t), q^{2}(t), \dot{q}^{l}(t), \dot{q}^{2}(t), l^{l}(t), l^{2}(t)\right)$ on corresponding products of $\Delta_{q} \times s_{q} \times \lambda$. For convenience the supersiripts "j" will be dropped until they are needed to avoid ambiguity.

Considering the links elastic we intruduce the deformation coordinates for the $i$-th 1 ink as shown in Fig. 1 , while using the Ritz-Kantorovitch series expansion 


$$
r_{i}\left(y_{i}, t\right)=\sum_{v=1}^{m} r_{i}^{\nu}\left(y_{i}\right) r_{i}^{\nu}(t)=r_{i}\left(y_{i}\right) r_{i}(t)
$$

and for $v_{i}\left(y_{i}, t\right), w_{i}\left(y_{i}, t\right)$ analogously, with the exact solution expected for m $\rightarrow$. We take large

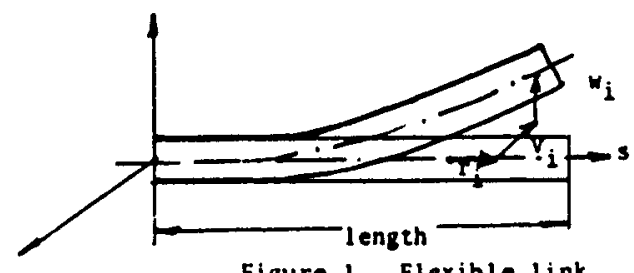

Figure 1. Flexible link

enough so that the Kantorovitch Iinearization is physically justified. The technical way about it is to stepwise subdividing the links between grid as long as the difference of results for successive 's becomes small. Having specified (2) we form the vector $n_{i}(t) \Delta\left(n_{1}(t), \ldots, n_{n}(t)\right)^{T}$, where $\eta_{1}(t) \triangleq\left(r_{i}(t), v_{i}(t), w_{i}(t)\right)^{T}$ and following [j] write the hybrid system as

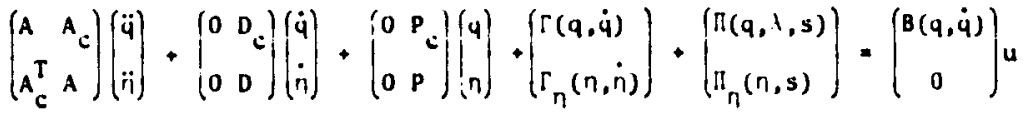

where $A_{\eta}(\eta, s), \Gamma_{\eta}(\eta, \dot{\eta}), n_{\eta_{l}}(\eta, s)$ are the elastic correspondents of $A, \Gamma, \| l$, while $A,(q, \eta)$, $D_{c}(q, \dot{q}, \eta, \dot{\eta}), P_{c}(q, \eta)$ and the internal damping $D(q, q, \eta, \eta)$ as well as the hybrid restoring coefficients $P(q, n)$ are matrices coupling the elastic and joint coordinates. These matrices are formed by integrals over tha shape functions, see $[5]$. Letting

$$
A(q, n, s)=\left(\begin{array}{ll}
A & A_{c} \\
A_{c}^{T} & A_{I 1}
\end{array}\right)
$$

to be the hybrid inertia matrix which is nonsingular positivo definite, we inertially decouple (3):

$$
(\ddot{4}, i i)^{T}+D(4, \dot{q}, n, \dot{r}, 1, s)+P(4, n, \lambda, s)=B(4, \dot{q}, 5) u
$$

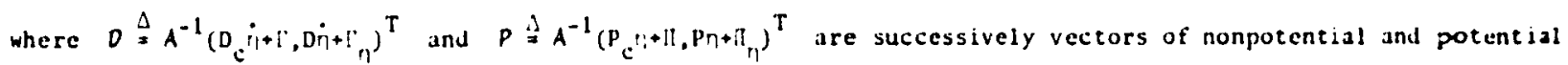
forces and the meaning of th: matrix 8 is ubvious. The vectors $4, \dot{\varphi}, 7$, $\dot{\eta}$ form the stats vector

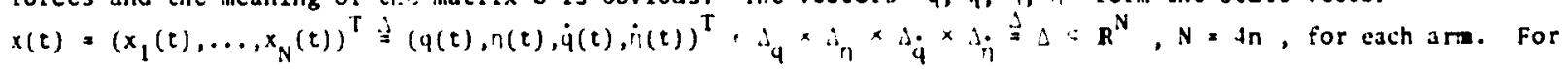
convenience (4) may be then written in the general state form

$$
\dot{x}=f(x, u, j, s)
$$

with $f=\left(f_{1}, \ldots, f_{N}\right)$ of the shape specified by (4) in an obvious way. Formally (5) may be written in the contingent form:

$$
\dot{x}=|f(x, u, t, s)| s * s:
$$

which for suitable $f(\cdot), P(\cdot), l(\cdot)$ has solutions $x(t)=k\left(x^{0}, t\right), t=0$, absolutely continuous curves through each $x^{0}=x(0)$ in $\Delta$. We shall consider the class of such solutions $k\left(x^{0}\right)$ by exhausting al 1 values ot $s(t)$ in (5) at each $t$.

4. The Reference Model

We let the given Cartesian "world" coordinates representation of the reference inodel in general terms

$$
\vdots_{m}=F\left(\lambda_{n}\right)
$$

with in $D O F, F_{z}(t) \in R^{3.2 n}$, and $F\left(l_{m}\right)$ suitable matrix, be off-line recalculated to the joint coordinate format of the rigid linear system

$$
\ddot{u}_{m}+D_{m}\left(u_{m}\right) \dot{q}_{m}+P_{m}\left(r_{m}\right) q_{m}=0
$$

with the $2 n$-vectors $\varphi_{m}, \dot{\varphi}_{m}$ of joint coordinates and velocitics, state $x_{m}(t)=\left(\varphi_{m}(t), \dot{\varphi}_{m}(t)\right)^{T} \cdot i=R^{r}$, and 


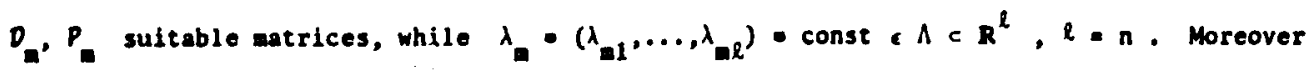

$$
P\left(\lambda_{a}\right)\left(q^{\circ}, n^{\circ}\right)=0
$$

with $\left(q^{*}, n^{e}\right)$ denoting the equilibria of $(3)$ on the surface $\dot{q}=0, \dot{\eta}=0$. The total energy of the model will be denoted by $E_{m}\left(\xi_{m}, \xi_{m}\right)$ in the world coordinates and $E_{m}\left(q_{m}, q_{m}\right)$ in the joint coordinates, obviously equal to one another. Then

$$
E_{m}\left(q_{m}, \dot{q}_{m}\right)=l \dot{q}_{m}^{T} \dot{q}_{m}+\int_{q_{m}}^{q_{m}} p_{m}\left(\lambda_{m}\right) d \sigma
$$

and substituting (7),

$$
\dot{E}_{m}\left(q_{m}, \dot{q}_{m}\right)=-D_{m}\left(\lambda_{m}\right)\left(\dot{q}_{m}\right)^{2}
$$

The model is selected such as to allow achieving of a stipulated target behavior in the state space. To focus attention on something specific and yet general enough, let it bo stability of the origin, guaranteed by the nonaccummulation of the total energy i.e. non-negative damping

$$
\dot{E}_{m}\left(q_{m}, q_{m}\right) \leq 0, v \dot{q}_{m} \neq 0
$$

while

$$
\nabla E_{m}\left(q_{m}, \dot{q}_{m}\right)>0
$$

in-the-large i.e. on sane $c \Delta_{L}=\Delta-\Delta_{L}$, where $\Delta_{L}$ is the set in $R^{N}$ enclosing all the equilibria.

5. Objectives

Now we consider both arms $j=1,2$ and the model together. The block schere of the system is shown in Fig. 2.

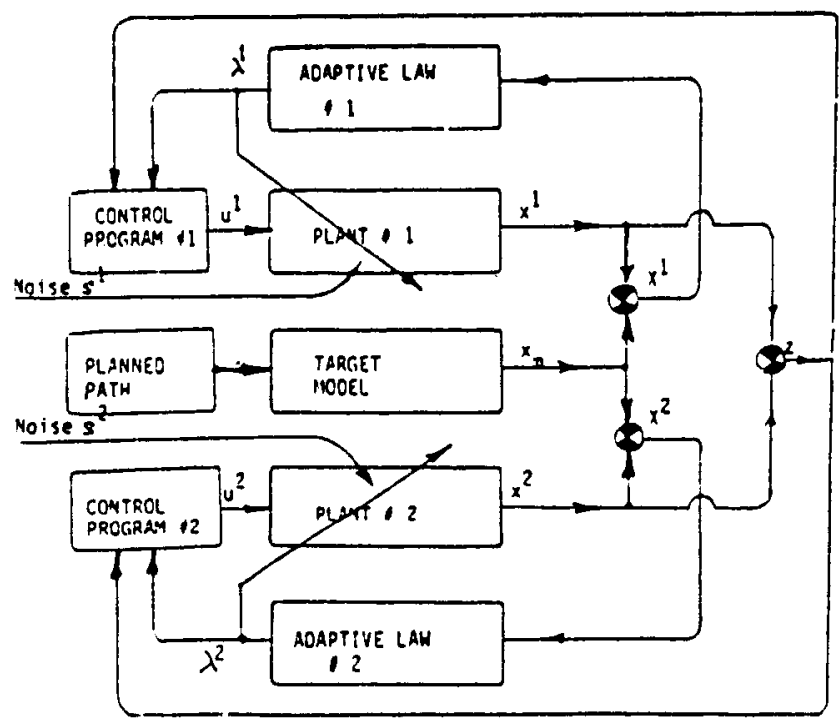

Figure 2. Block scheme of the system

Define two product $2 N$-vectors $x^{j}(t)=\left(x^{J}(t), x_{m}(t)\right)^{T}, \Delta x \Delta \dot{\xi} \Delta^{2}$ and two 2 -vectors $x^{j}(t)=\lambda^{j}(t)-\lambda_{m}$. which vary in $\Delta^{2} x_{i j}$ generating the product trajectories $\left(x^{j}\left(x^{j o}, t\right), x^{j}\left(x^{j 0}, t\right)\right), t \geq 0, x^{j 0}=x^{j}(0)$, $x^{j 0}=x^{j}(0)$. Then we define the "diagonal" sets

$$
M^{j}=\left(\left(x^{j}, x^{j}\right) \times J^{2} x_{i} \mid x^{j}=x_{m}, x^{j}=0\right\}, j=1,2,
$$

and given stipulated $\mu^{j}>0$, their neighbourhoods

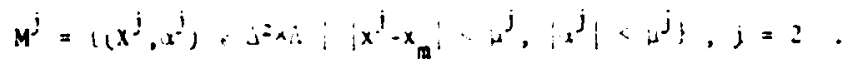

Morenver we let $i_{0}$ be a desired subset of $i$ where we want the tracking to occur, and let $t_{c}$ be the 
stipulated tise after which the tracking is attained with eccuracy $\mu^{j}$.

pirst Objective: The manipuletor arms (1) are mutuaily $\mu$-tracking the target (7) on $\Delta_{0}$ if thero is a pair of controllers $p^{j}(\cdot), j=1,2$ such that for each solution $k^{j}\left(x^{j 0}, t\right), t \geq 0$ of $(4)$ in $K\left(x^{j o}\right)$, the set $\Delta_{\rho}^{2} \times \Lambda$ is positively invariant: $\left(x^{j 0}, \alpha^{j o}\right), \Delta_{0}^{2} \times \Lambda-\left(x^{j}(t), \alpha^{j}(t)\right) \subset \Delta_{0}^{2} \times \Lambda$ and given $t_{c}$, for each $K(\cdot) \in K\left(x^{j o}\right)$ the product trajectorles satisfy

$$
\left(x^{j}(t), a^{j}(t)\right), M_{\mu}^{j}, v t \geq t_{c} .
$$

The convergence is illustrated in Fig. 3.

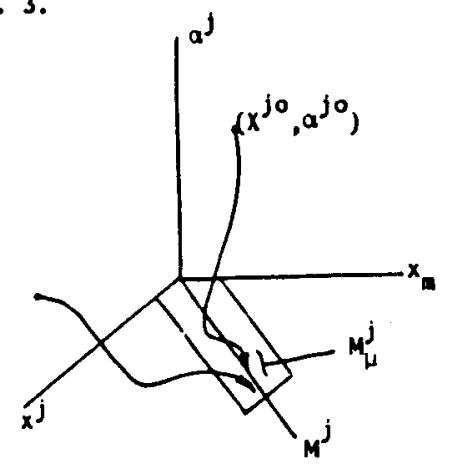

Figure 3. Convergence of product trajectories

Suppose the transformation from joint to world coordinates (forward kinematics) is given by

$$
\xi_{j}^{j}=e_{j}^{j}\left(q^{j}, n^{j}\right), c=1, \ldots, 3 \cdot 2 n
$$

and denote $z(t) \Theta\left(x^{2}(t), x^{2}(t)\right)$. Then we let the set

$$
A \Delta\left\{z=\Delta^{2}|| \xi_{j}^{1} \cdot \xi_{v}^{2} \mid-d, v o, v=1, \ldots, 3 \cdot 2 n\right\}
$$

be the collision set between arms to be avoided. We define $c A=\Lambda_{0}^{?}-A$, specified by $\left|\varepsilon_{0}-\xi_{1}^{2}\right|>d$, and let

$$
\Delta_{A} \Delta\left\{z \cdot \Delta^{2}|d<| \xi \xi_{0}^{4}-\xi_{j}^{2} \mid<L\right\}
$$

be the "slow down" safety zone, with $i>0$ suitable constant.

Second objective: The tracking arms (1) avoid collision iff there is $\Delta_{A}$ such that for any $z^{0}$. CA, and any pair $k^{j}(\cdot) \in K^{j}\left(x^{j o}\right)$ the corresponding product trajectory.

$$
z\left(z^{0}, t\right) \cdot C A, \quad v t>0 \text {. }
$$

\section{Sufficient Conditions}

We return now to the first objective and specify by $N\left[j\left(a_{0}^{2} \times \Lambda\right)\right]$ a neighborhood of the boundary $;\left(\therefore_{0}^{2} \times 1\right)$

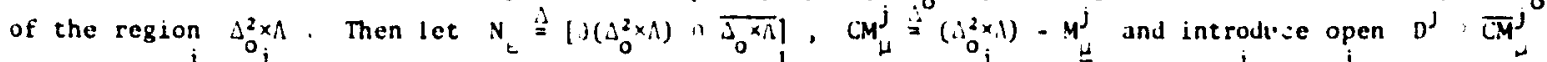
such that $D^{j} \cap \mathbb{M}^{j}=6$. Further we consider four $c^{1}$-functions $v_{s}^{j}(\cdot): \frac{\vec{v}}{b}, R, v^{j}\left(1: 0^{j}, R, j=1,2\right.$ with the positive constants

$$
\begin{aligned}
& v_{s}^{j}=v_{s}^{j}\left(x^{j}, s^{j}\right),\left(x^{j}, s^{j}\right), ;\left(i \times x_{\Lambda}\right) \\
& v_{\mu}^{j-}=\inf v_{j}^{j}\left(x^{j}, x^{j}\right) \mid\left(x^{j}, x^{j}\right) \cdot M_{j}^{j} \cap \overline{C M}_{j}^{j} \\
& v_{j}^{j+} \cdot \sup v_{, j}^{j}\left(x^{j}, x^{j}\right) \mid\left(x^{j}, x^{j}\right) \cdot\left(\left(.^{2} \times, \lambda\right), \mathrm{CM}^{j}\right)
\end{aligned}
$$

The first relation obviously requires forming $v_{S}^{j}(\cdot)$ from suitable,$\left(j_{0} x_{1}\right)$ taken as its level, or conversely, forming $3 \Delta, 3 A$ from levels of suitable $V_{S}(\cdot)$. In the latter iase $a^{\circ} i_{0} . A$ smallur than these desired will be the secule choice.

THEOREM 1: Objective 1 is attained if, given $\therefore_{0}, \therefore, 4$ there are prog-ans $p^{j}(\cdot)$ and functions $v_{S}^{j}(\cdot), v_{j}^{j}(\cdot)$ such that for $a l l\left(x^{j}, x^{j}\right), \$_{j}^{2} \times a$. 


$$
\begin{aligned}
& \text { (i) } \quad v_{s}^{j}\left(x^{j}, a^{j}\right) \leq v_{s}^{j}, v\left(x^{j}, a^{j}\right), N_{\varepsilon}, j \cdot 1,2 \\
& \text { (ii) for each } u^{j} \in p^{j}\left(x^{4}, x^{2}\right) \text {; } \\
& v_{s}^{j}\left(x^{j}(t), a^{j}(t)\right)<0, v_{s}^{j}, s \\
& \text { along the product trajectories }\left(x^{j}\left(x^{j 0}, t\right) a^{j}\left(a^{j 0}, t\right)\right), t 20, j-1,2 \text {; } \\
& \text { (iii) } \quad 0<v_{\mu}^{j}\left(x^{j}, a^{j}\right) \leq v_{\mu}^{j+}, v\left(x^{j}, a^{j}\right), \overline{O M}_{\mu}^{j}, j=1,2 \text {; } \\
& \text { (iv) } \quad v_{\mu}^{j}\left(x^{j}, a^{j}\right) \leq v_{\mu}^{j-}, v\left(x^{j}, a^{j}\right) \in D^{j} \cap M_{\mu}^{j}, j=1,2 \text {; } \\
& \text { (v) for each } u^{j}=p^{j}\left(x^{4}, x^{2}\right) \text { there is a constant } c_{j}>0 \text { such that } \\
& \dot{v}_{\mu}^{j}\left(x^{j}(t), a^{j}(t)\right) \leq-c_{j}, v_{s}^{j} \in S
\end{aligned}
$$

Remark 1: The Objective 1 holds after a stipulated $t_{c}<\infty$ if Theoren 1 is satisfied with $c_{j}$ solected by

$$
c_{j} \Delta \frac{\Delta v_{\mu}^{j+}}{t_{c}}, j=1,2 .
$$

THEOREM 2: Objective $Z$ is attained if thoorem 1 holds and given $d$ there is a $C^{l}$-function $V_{A}(\cdot): \dot{C}_{A} \rightarrow R$ such that for the tracking pair $\mathrm{p}^{\mathrm{j}}(\cdot)$, for all $\mathrm{Z} \cdot \mathrm{CA}$,

(vi) $V_{A}(z)>V_{A}(z), V_{z} \in$ iA ;

(vii) for each $u^{j} \bullet p^{j}(z)$.

$$
\dot{v}_{A}\left(z\left(z^{0}, t\right)\right)=0, z^{0} \in \Delta_{A}, v_{3}^{j}, s
$$

along product trajectories $z\left(z^{0}, t\right), t \geq 0$.

PROOF. Suppose some $z\left(z^{0}, t\right), t \geq 0, z^{0} \in \Delta_{A}$ crosses $3 A$ at $t_{1}>0$. Then by $(v i), v_{A}\left(z\left(t_{1}\right)\right)<V_{A}\left(z^{0}\right)$ which contradites (vii).

7. Controllers and Adaptive Laws

l.et us set up

$$
\begin{aligned}
& V_{S}^{j} \stackrel{A}{E} E_{i A}\left(x^{j}\right)+E_{m}\left(x_{m}\right)+a^{j} x^{j} ; \\
& v_{\mu}^{j} \therefore\left\{\begin{array}{l}
\mid E_{m}\left(x^{j}\right)-E_{m}\left(x_{m}\right)+a^{j} a^{j},\left(x^{j}, x^{j}\right), C M_{j}^{j} \\
a^{j} x^{j},\left(x^{j}, x^{j}\right) \cdot M_{\mu}^{j}:
\end{array}\right. \\
& v_{A}=\operatorname{lit}\left(x^{4}\right)-E_{m}\left(x^{2}\right) \mid
\end{aligned}
$$

where $a^{j}=\left(\operatorname{sign} x_{1}^{j}, \ldots, \operatorname{sign} a_{n}^{j}\right), j=1,2$, and $E_{x}\left(x^{j}\right)$ is $E_{n}(\cdot)$ with $x_{m}$ exchanged for $x^{j}$. Choosing $N_{\text {- I }} \mathrm{C}_{l}$. , the character of $E_{m}(\cdot)$ specified additionally by (12), satisfies (i), (iii) and (iv).

To see that $\left(v_{1}\right)$ holds, observe that $E_{m}\left(x^{j}\right)=E_{m}\left({ }^{j}, j\right)$ of $(6)$ and that increasing the distance $i \zeta_{\nu}-i_{j}^{2} ;=0$ for at least one $\mathcal{f}$ from its of value increases the value of $v_{A}$.

To check upon conditions (ii), (v), (vii) we differentiate (21) - (23) with respect to time

$$
\begin{aligned}
& \dot{v}_{S}^{j}(t)=\dot{E}_{\pi}\left(x^{j}\right)+E_{a}\left(x_{n}\right)+a^{j} i^{j} ;
\end{aligned}
$$

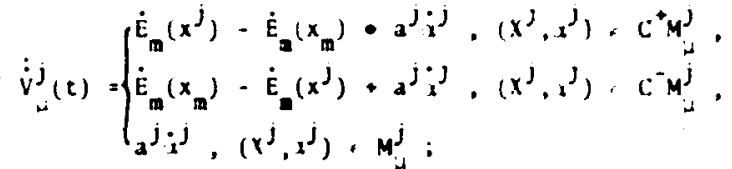

$$
\begin{aligned}
& \dot{V}_{A}(t)=\left[E_{m}\left(x^{4}\right)-E_{m}\left(x^{2}\right)\right] \cdot\left[\dot{E}_{m}\left(x^{4}\right)-\dot{E}_{m}\left(x^{2}\right)\right] \text {. }
\end{aligned}
$$

where

$$
\dot{H}_{m}\left(x^{j}\right)=E_{m}\left(x^{j}\right) \cdot f^{j}\left(P^{j}, u^{j}, i^{j}\right)=\left(B u-D-p+p_{m} y_{m}\right)(\dot{y}, \dot{i})
$$


The brackets of the functions $B, D, P$ dropped for clarity. Moreover $c^{t} w_{\mu}^{j}$ are subsets of $O_{\mu}^{j}$ defined by

$$
\begin{aligned}
& C^{+} \mu_{\mu}^{j}: E_{E}\left(x^{j}\right) 2 \ddot{\varepsilon}_{\mathbb{E}}\left(x_{j}\right) \\
& C^{-} \mu_{\mu}^{j}: E_{m}\left(x^{j}\right)<E_{m}\left(x_{m}\right) \text {. }
\end{aligned}
$$

with a suitable choice of initial states the following set of conditions iaples (ii), (v) and (vii):

(a) $\min _{j} \max _{j} \dot{E}_{m}\left(x^{j}\right)=\dot{E}_{m}\left(x_{m}\right), r\left(x^{j}, a^{l}\right), c^{+} m_{\mu}^{j}$.

$$
\underset{u^{j}}{\max } \sin _{s^{j}} \dot{E}_{m}\left(x^{j}\right) \geq \dot{E}_{m}\left(x_{m}\right), r\left(x^{j}, a^{j}\right) \in c^{-} m_{\mu}^{j} ;
$$

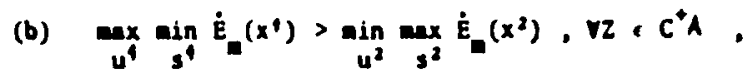

$$
\min _{u^{4}} \max _{s^{1}} \dot{E}_{m}\left(x^{4}\right)<\max _{u^{2}} \min _{s^{2}} \dot{E}_{m}\left(x^{2}\right), v z \cdot c^{-1} \text {. }
$$

for $\dot{q} 0, \dot{n} \neq 0, j=1,2$. In the above $c^{2} A$ are subsets of $d A$ defined by:

$$
\begin{aligned}
& c^{+} A: E_{m}\left(x^{4}\right) \geq E_{m}\left(x^{2}\right), \\
& C^{-} A: E_{m}\left(x^{4}\right)=E_{m}\left(x^{2}\right) .
\end{aligned}
$$

(c) $a^{j} \dot{i}^{j}=\dot{E}_{a}\left(x_{a}\right) \cdots j, a^{j} \neq 0, j=1,2$.

Observe that for $x^{j}=0$ there is no need for aduptation and that the systen (4) crosses the surface $\dot{4}$. 0 . $\dddot{V}_{1}-0$ time instantenously (vertically) so there is no need for controf in view of the smoothness of trajectories. Conditions (a), (b) are called control conditions helping to design p (.), condition (c) is called adaptive, helping to design adaptive laws. Let us check that (a), (b), (c) indeed imply ( $i i)$, (v), (vii). Consider first

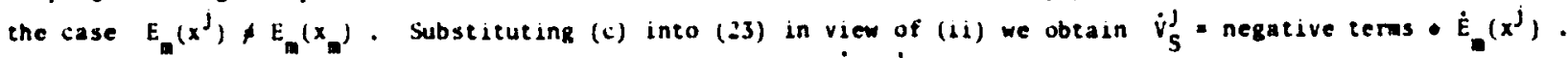
Boundediness of the work space necessitates the power: $\dot{F}_{w}\left(x^{J}\right) \leq 0$ thus (1b). Substituting (a), (c), and (11) into (2t) with (18, we satisfy (v) in stipulated time $t_{c}$. Note that this holds for any initial states. The case $E_{m}\left(x^{j}\right)=E_{m}\left(x_{m}\right)$ is trivial as then $\dot{V}_{S}^{J}-3 \dot{E}_{m}\left(x_{m}\right) \cdot 0, \dot{v}_{j}=\dot{E}_{m}\left(x_{m}\right)-c_{j}-c_{j}$. Finally we check (vit). Again first let $E_{m}\left(x^{4}\right) \neq E_{m}\left(x^{2}\right)$ and observe that $(b)$ substituted to $(20)$ Implies (vii). The case $\dot{E}_{m}\left(x^{0}\right)=\dot{L}_{n}\left(x^{2}\right)$ is obviously trivial.

Observe that, with (11), (c) is implied by the following adaptive laws

$$
i_{1}^{j}=-\operatorname{sign} i_{i}^{1}\left(D_{m i} \dot{q}_{m i}^{2}-\frac{i}{n}\right) \text {. }
$$

for $t^{j} \neq 0,1=1, \ldots, n$. Physically the solutions $i^{j}\left(s^{j 0}, t\right)$ represent the model energy flux which become positsie or negative depending upon where, "jo is located (below or above the surface s) 0 ) thus regulating the increment of $j$ to $=0$ ro irom anywliere uutside the surface? 0 .

8. Modular Double RP-Manipulator Fig. 4 .

Our technique is lllustrated below on the case study of the four nof manipulator with two drms shown in

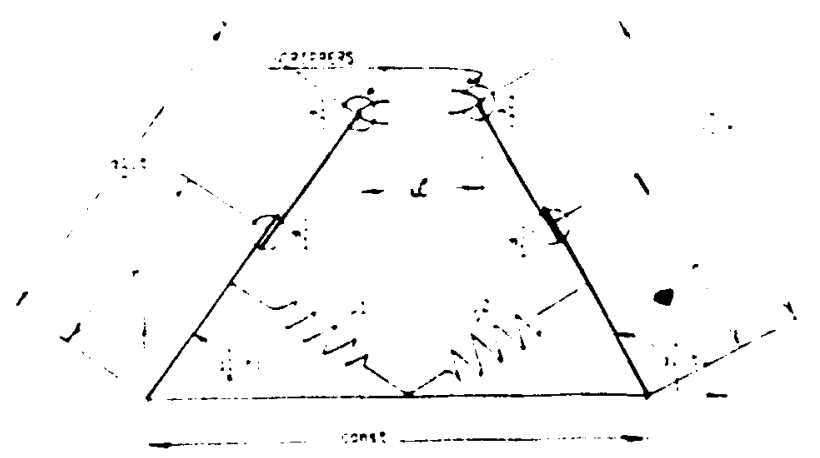

ligure t. The modular :-Rp sanipulitior 
The lagrange equetions of sotion for each arm result in the following wotion equetions

$$
\begin{gathered}
\left(m_{1} r^{2}+m_{2} q_{2}^{2}\right) \ddot{q}_{1}+2_{-} q_{2} \dot{q}_{1} \dot{q}_{2}+\lambda_{3}\left|\dot{q}_{1}\right| \dot{q}_{1}+z_{1}\left(m_{1} r+m_{2} q_{2}\right) \cos q_{1}-m_{1} g r+\lambda_{1} q_{1}+{ }_{2} q_{1}^{3}-u_{1} \\
q_{2} \ddot{q}_{2}-m_{2} q_{2} q_{1}^{2}+\lambda_{4} q_{2}+m_{2} \sin q_{1}=u_{2} .
\end{gathered}
$$

Here $\lambda_{3}, \lambda_{4}$ are daping coefficients, $\lambda_{1}, \lambda \lambda_{2}$ spring coefficients, s-gravity acceleration, the reainder of notetions shown in Fig. 4. The superscripts "f", $f-1,2$, are ignored for the tine being. ive take the possible payload on the grippers as unknow but within known bounds which sakes ${ }_{2}$ specified by

$$
\pm s a_{2} \leq \bar{a} \text {. }
$$

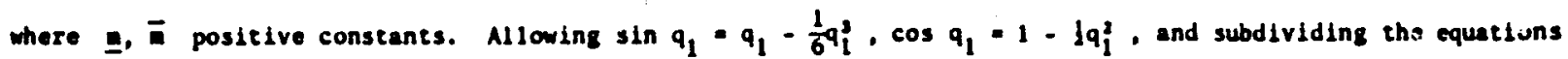
(29) by corresponding inertis coefficients we obtain:

$$
\ddot{q}_{i} \cdot r_{1}+n_{i} \cdot u_{i}, i=1,2
$$

where

$$
\begin{aligned}
& r_{1}=\frac{2 m_{2} q_{2} \dot{q}_{1} \dot{q}_{2}+\lambda_{3}\left|\dot{q}_{1}\right| \dot{q}_{1}}{m_{1} r^{2}+m_{2} q_{2}^{2}}, \\
& r_{2}=-q_{2} \dot{q}_{1}^{2}+1 / m_{2} \lambda_{4} \dot{q}_{2}, \\
& n_{1}=\frac{1_{1} q_{1}-18 m_{1} r q_{1}^{2}+1_{2} q_{1}^{3}-18 m_{2} q_{2} q_{1}^{2}+g_{2} q_{2}}{-m_{1} r^{2}+a_{2} u_{2}^{2}} \\
& \pi_{2}=8 q_{1}-\frac{1}{6} q_{1}^{3}, \\
& B_{1}=\frac{1}{m_{1} r^{2}+m_{2} q_{2}^{2}} \cdot B_{2}=\frac{1}{a_{2}}
\end{aligned}
$$

The reference model is taken as

$$
\left.\begin{array}{l}
\ddot{q}_{m 1}+b_{33} \dot{q}_{m 1}+1_{n 1} q_{m 2}+8 q_{n 2}=0 \\
\ddot{q}_{m 2}+\lambda_{m 4} \dot{u}_{m 2}+84_{m 1}=0 .
\end{array}\right\}
$$

The total energy uf the model is

$$
E_{m}\left(u_{m} \cdot \dot{q}_{m}\right)=i\left(\dot{q}_{m 1}^{2}+\dot{q}_{m 2}^{2}\right)+i \alpha_{m 1} u_{m 1}^{2}+28 q_{m 1} u_{m 2} \text {. }
$$

nifferentlating it with respect to time and substituting (32),

$$
\dot{E}_{m}\left(q_{m} \cdot \dot{q}_{m}\right)=-i_{m 3} \dot{u}_{m 1}^{2}-\lambda_{m 4} \dot{q}_{m 2}^{2} .
$$

Accordingly,

$$
\dot{f}_{m}(q, \dot{u}) \cdot\left(B_{1} u_{1}-i_{1}\right) \dot{u}_{1} \cdot\left(B_{2} u_{2}-v_{2}\right) \dot{u}_{2}
$$

Choose $\left(x^{j 0}, x^{j 0}\right), C^{+} M^{J}, y=1,2$ and $z^{0}, C^{+} A$. Then the control conditions (a), (b) hold if successively

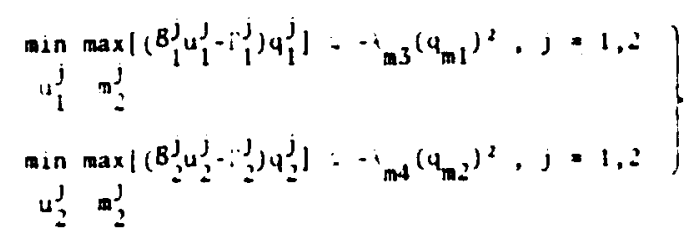

and 


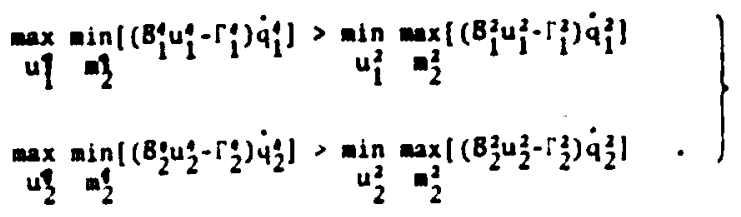

Thus we choose $u_{i}$ such that for $\dot{q}_{i} \neq 0$.

$$
\min _{u 1} \max _{m}\left(\left(B_{i} u_{i}^{q}-r_{i}\right) \dot{q}_{1} l=-\lambda_{m 3}\left(\dot{q}_{m 1}\right)^{2}\right.
$$

and for such $u_{i}$, we choose $u_{i}^{2}$ sat isfying

$$
\min _{u_{1}^{2}} \max _{1}^{2}\left[\left(B_{1}^{2} u_{1}^{2}-\Gamma_{1}^{2}\right) \dot{q}_{1}^{2}\right]<\max _{u \mid} \min _{2}^{q}\left[\left(B_{i}^{q} u_{1}^{4}-\Gamma_{1}^{q}\right) \dot{q}_{1}^{q}\right]
$$

The procedure for $u_{2}^{t}$ and $u_{2}^{2}$ is identical utilizing the second inequalities of (34), (35). Assuming symatry of arms: $m_{1}^{4}=m_{1}^{2}=m_{1}, m_{2}^{4}=m_{2}^{2}=m_{2} \in\left[m_{,}, \bar{m}\right], r^{4}=r^{2}, r$, and substituting the expressions for $r_{i}^{j}, Z_{i}^{j}, B_{i}^{j}, i, j=1,2$, we obtain the tracking controllers

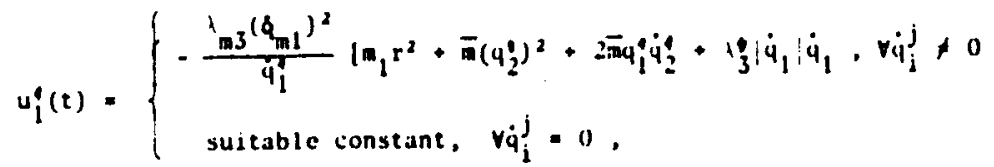

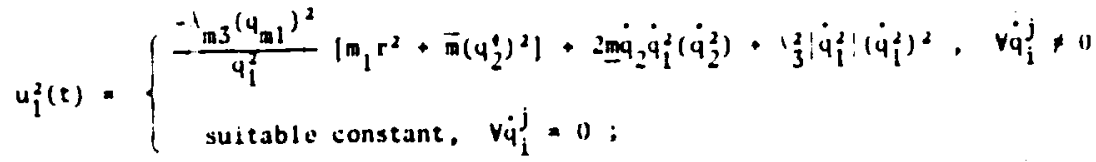

and the collision avoidance controllers

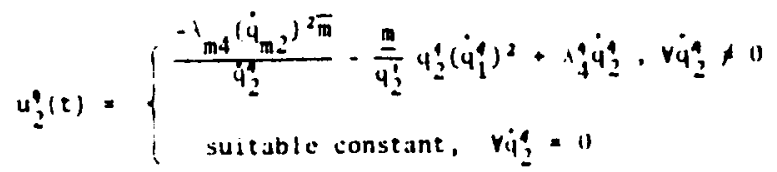

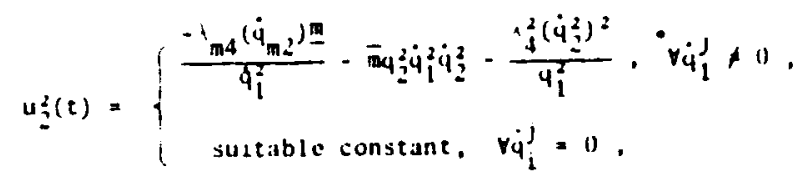

which imply the control conditions (a), (b) for our example. The ajaptive laws (2t) are

$$
\begin{aligned}
& i j 0, \quad j-0 \\
& i_{3}^{j}=-\left(\operatorname{sign} x_{3}^{j}\right) t_{m} \dot{4}_{m 1}^{2}-i c, \\
& \dot{i}=-\left(\operatorname{sign} a_{f}^{j}\right){ }_{m 4} \dot{4}_{m 2}^{2}-i c
\end{aligned}
$$

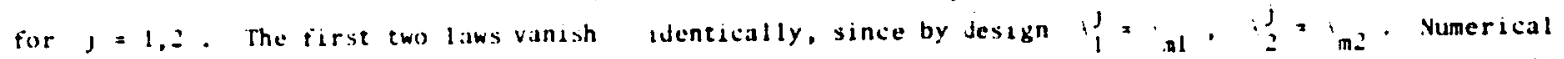
simulation of our nodulur case, with the datia $m_{1}=70 \mathrm{~kg}, \underline{m}=30 \mathrm{~kg}, \overline{\mathrm{m}}=40 \mathrm{~kb}, \mathrm{r}=11.60 \mathrm{~m}, 1 \mathrm{ml}=20$, $1 \mathrm{mz}=20$, $i_{3}=5$, ind $=2$, 15 shown in $l i g .5$, and confirms the convergence-jvoidance required.

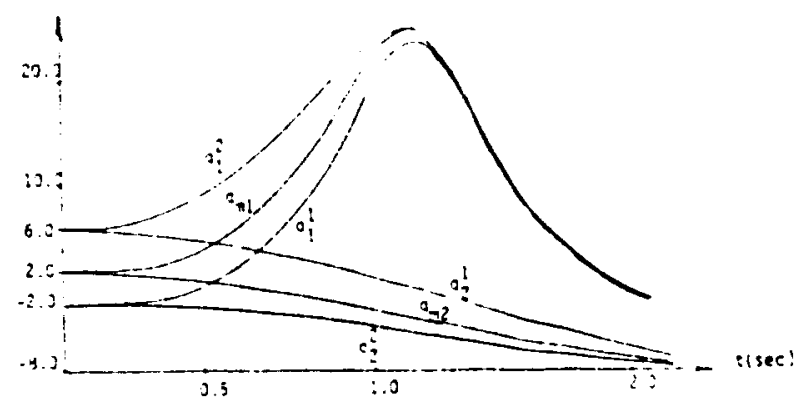

Figure 5. Numerical staulation 


\section{References}

[1] J.M. Skowronski, Control Dynanics of Robot Manipulators, Academic Prdss, 1960.

[2] J.M. Skowronsk1, Model Reference Adaptive Control Under Uncertainty of Nonlinear Flexible Manipulators, AIM Paper 186 - 1976 - CP, Proc. AIM Guidance, Navigation and Control Conf., Williamsburg.Vr. 1986.

[3] G. Leitann, J.M. Skowronski, On Avoidance Control, Journal of Optiaization Theory and Applications, Dec. 1977.

(4) G. Leitmann, J.M. Skowronski, A Note on Avoldance Control, Optiaal Control Applications and Meţhods, Dec. 1983.

[5] A. Truckenbrodt, Effects of Elasticity on the Performance of Industrial Robots, Proc. 2nd IASTED Danos International Symposium on Robotics, Switzerland, 1982, pp. 52-56. 